\title{
Tuberculosis burden in stage 5 chronic kidney disease patients undergoing dialysis therapy at Livingstone Hospital, Port Elizabeth, South Africa
}

\author{
S Ndamase, ${ }^{1}$ MB ChB, FCP (SA); I Okpechi, ${ }^{2}$ MBBS, FWACP, PhD, Cert Nephrology (SA) Phys; H Carrara, ${ }^{3}$ MPH; \\ J Black, ${ }^{4}$ MB ChB, FCP (SA), Cert ID (SA); G Calligaro, ${ }^{5}$ BSc Hons (Phys), MB BCh, Dip PEC (SA), MMed (Internal Medicine), \\ FCP (SA), Cert Pulmonology (SA); R Freercks, ${ }^{2}$ MB ChB, FCP (SA), Cert Nephrology (SA) Phys, MPhil, FRCP (Lon)

\begin{abstract}
${ }^{1}$ Department of Medicine, Faculty of Health Sciences, University of Cape Town and Groote Schuur Hospital, Cape Town, South Africa ${ }^{2}$ Division of Nephrology and Hypertension, and Kidney and Hypertension Research Unit, Department of Medicine, Faculty of Health Sciences, University of Cape Town, South Africa

${ }^{3}$ Department of Public Health and Family Medicine, Faculty of Health Sciences, University of Cape Town, South Africa

${ }^{4}$ Division of Infectious Diseases and HIV Medicine, Department of Medicine, Faculty of Health Sciences, University of Cape Town, South Africa
\end{abstract} \\ ${ }^{5}$ Division of Pulmonology, Department of Medicine, Faculty of Health Sciences, University of Cape Town, South Africa
}

Corresponding author: S Ndamase (cvindamase@gmail.com)

Background. Tuberculosis (TB) is currently the leading cause of death from a single infectious agent worldwide. Patients who receive dialysis are particularly vulnerable to TB infection owing to immune dysfunction. Nonetheless, there is a paucity of incidence data on dialysis patients infected with TB in high-burden countries, such as South Africa (SA).

Objectives. To determine the incidence of $\mathrm{TB}$ in prevalent chronic kidney disease stage 5 (CKD-5D) patients on dialysis at a single centre in Eastern Cape Province, SA, and to identify the risk factors associated with TB infection.

Methods. We conducted a retrospective cohort study of all consenting CKD-5D patients between April 2010 and March 2014 at Livingstone Hospital Renal Unit, Port Elizabeth, the Eastern Cape. TB was defined as definite or probable according to World Health Organization (WHO) criteria, and the cohort was split into those who developed $\mathrm{TB}(\mathrm{TB}+)$ and those who did not (TB-).

Results. One hundred and eleven patients were enrolled - predominantly black Africans (73\%) and women (53\%); the mean age (standard deviation (SD)) was 42 (9.8) years. The prevalence of HIV infection was 11\%, all patients were receiving antiretroviral treatment and all had suppressed viral loads. Sixty-eight patients were on haemodialysis and 43 on peritoneal dialysis. Nineteen patients were diagnosed with 20 episodes of TB; 14 cases were pulmonary TB and 6 cases extrapulmonary TB. Of the patients with TB, 2 were HIV-infected, 7 (35\%) were definite TB cases and $13(65 \%)$ were probable cases. The calculated incidence rate was $4505 / 100000$ patient years. Only informal housing $(30 \%$ in TB+ v. $12 \%$ in TB-; $p=0.042)$ and a history of hospitalisation $(90 \%$ v. $76 \%$, respectively; $p=0.042)$ were significantly associated with a diagnosis of $\mathrm{TB}$.

Conclusions. Dialysis patients in the Eastern Cape region of SA are at extremely high risk of acquiring TB, with an incidence rate 4.1 times that of the local population and $>5$ times that of the general SA population. Only informal housing and a history of hospitalisation were identified as positive risk factors for TB in this young population with a low HIV prevalence. Isoniazid prophylaxis in this high-risk group might be of benefit, but further studies are required to inform such treatment.

S Afr Med J 2020;110(5):422-426. https://doi.org/10.7196/SAMJ.2020.v110i5.14035

Tuberculosis (TB), caused by the organism Mycobacterium tuberculosis, remains a major global health burden and is responsible for ill health among millions of people each year. It is currently the leading cause of death as a result of a single infectious agent worldwide. ${ }^{[1]}$ South Africa (SA) has a reported TB incidence of 567/100 000 cases per year. ${ }^{[1]}$ The reported incidence of TB in the Nelson Mandela Bay district, Eastern Cape Province, SA, is $1009 / 100000$ cases per year ${ }^{[2]}$ which is much higher than the national average. In the Eastern Cape, it is reported to be the leading cause of death in persons $25-64$ years of age. ${ }^{[2]}$ Unfortunately SA, where the treatment cure rate is $82 \%$, is failing at a national level to reach the proposed World Health Organization (WHO) target of $90 \% .^{[1]}$

Patients who receive dialysis are particularly vulnerable to active $\mathrm{TB}$ infection compared with the general population, due to immune dysfunction induced by their uraemic state. ${ }^{[3-5]}$ In other low- and middle-income countries (LMICs), the incidence of TB among prevalent dialysis patients is 6.9 - 52.5 times higher than in the general population, depending on regional factors and background prevalence. ${ }^{[6-8]}$ Other factors that might contribute to decreased immunity are malnutrition, vitamin $\mathrm{D}$ deficiency and hyperparathyroidism. ${ }^{[9]}$

There is a paucity of data regarding the incidence of $\mathrm{TB}$ in SA dialysis patients, where the background population prevalence is among the highest in the world. We are aware of only one published single-centre SA study by Tamayo-Isla et al., ${ }^{[10]}$ conducted in Polokwane, Limpopo Province, SA, where the authors describe a high mortality rate associated with $\mathrm{TB}$ peritonitis in continuous ambulatory peritoneal dialysis (CAPD) patients.

\section{Objectives}

To determine the incidence of TB in chronic kidney disease stage 5 (CKD-5D) patients receiving dialysis at a single centre in the Eastern 
Cape, SA, and to identify modifiable and non-modifiable risk factors associated with TB.

\section{Methods \\ Study design}

We conducted a retrospective cohort study of all consenting prevalent CKD-5D patients between April 2010 and March 2014 in the Livingstone Hospital Renal Unit in the Eastern Cape. A total of 111 consenting prevalent dialysis patients were enrolled in the study during the 4-year period; no patient had TB at enrolment.

\section{Data collection}

TB was defined as definite or probable according to WHO criteria. ${ }^{[11]}$ Definite TB patients were defined as those in whom TB complex was identified from a clinical specimen by culture or newer methods, including line-probe assays. Probable TB patients were those with constitutional symptoms, radiological features suggestive of TB and/or caseating granulomas on histological examination, but with no positive culture identification of TB. ${ }^{[11]}$ A medical questionnaire with all the required data was used. Patient demographics, including dialysis vintage, age, gender, race (self-reported), employment status, smoking, alcohol use, history of hospitalisation for any cause during the study period but prior to enrolment, housing type and TB household contact status were recorded. Details regarding the diagnosis of TB, mean time between initiation of dialysis and diagnosis, HIV status, vitamin D level, comorbidities and medications were also recorded.

\section{Statistical analysis}

The estimated incidence was calculated as the percentage of patients who developed TB during the study period. This incidence was then converted into an incidence per 100000 divided by 4 (for the 4 years) to give an estimated incidence per 100000 per year of study period.
Normality was determined with the Shapiro-Wilk test. Continuous variables were expressed as mean (standard deviation (SD)), while categorical variables were presented as frequencies and percentages. Univariate analysis was performed using the independent Student's $t$-test, $\chi^{2}$ test or Wilcoxon rank-sum test, as appropriate. All analyses were conducted using Stata 12.0 (StataCorp., USA).

\section{Ethical approval}

The study was approved by the Human Research Ethics Committee of the University of Cape Town, SA (ref. no. HREC/REF 127:2014), and permission for conducting the study was obtained from local authorities.

\section{Results}

Baseline characteristics of patients, including gender, HIV status, prior use of immunosuppressives, employment, smoking, known TB contact status and dialysis modality, are presented in Table 1. A total of 111 patients were enrolled, predominantly black Africans (73\%) and women (53\%); their mean age (SD) was 42.1 (9.8) years. The prevalence of HIV infection was $11 \%$, and all patients were receiving antiretroviral treatment. Sixty-eight patients received haemodialysis and 43 peritoneal dialysis; $93 \%$ were unemployed.

The characteristics of patients with TB are shown in Table 2. Nineteen (18\%) patients were diagnosed with TB over the 4-year study period, with 1 patient treated for TB on two occasions (\#6 and \#7, Table 2), giving a total of 20 TB cases; 14 patients had pulmonary and 6 extrapulmonary TB. Of the 20 TB patients (Fig. 1), 7 (35\%) were definite TB cases and $13(65 \%)$ had probable TB. Of the 7 definite cases, 1 patient (\#5) had TB peritonitis with dialysate effluent culture positive for drug-sensitive TB; 2 patients (\#7 and \#11) had TB adenitis and culture-confirmed drug-sensitive TB; 1 patient (\#1) had disseminated TB with a miliary pattern on a chest radiograph, a

\begin{tabular}{|c|c|c|c|c|}
\hline Variables & Patients $(N=111), \%^{*}$ & $\mathrm{~TB}+(n=19), \%^{*}$ & TB- $(n=92), \%^{*}$ & $p$-value \\
\hline Age, mean (years) & 42 & 42.4 & 41.8 & 0.868 \\
\hline \multicolumn{5}{|l|}{ Gender } \\
\hline Female & 53 & 45 & 55 & 0.396 \\
\hline \multicolumn{5}{|l|}{ Employment status } \\
\hline Unemployed & 93 & 90 & 93 & 0.584 \\
\hline \multicolumn{5}{|l|}{ Smoking status } \\
\hline$\geq 10$ pack-years & 16 & 10 & 18 & 0.405 \\
\hline$<10$ pack-years & 84 & 90 & 82 & 0.405 \\
\hline Hospitalisation & 79 & 90 & 76 & 0.042 \\
\hline \multicolumn{5}{|l|}{ Housing } \\
\hline Brick & 85 & 70 & 88 & 0.042 \\
\hline Shack & 15 & 30 & 12 & 0.042 \\
\hline Use of biomass fuel & 8 & 5 & 8.7 & 0.582 \\
\hline TB contact & 28 & 40 & 25 & 0.174 \\
\hline \multicolumn{5}{|l|}{ HIV status } \\
\hline Positive & 11 & 10 & 11 & 0.909 \\
\hline Use of immunosuppression & 8 & 1.4 & 6.6 & 0.171 \\
\hline \multicolumn{5}{|l|}{ Vitamin $\mathrm{D}, \mathrm{nmol} / \mathrm{L}$} \\
\hline Deficiency and insufficiency & 45.7 & 20.8 & 79.2 & 0.800 \\
\hline \multicolumn{5}{|l|}{ Dialysis modality } \\
\hline Haemodialysis & 61 & 18 & 82 & 0.898 \\
\hline Peritoneal dialysis & 39 & 19 & 81 & 0.898 \\
\hline
\end{tabular}




\begin{tabular}{llllllll}
\multicolumn{2}{l}{ Table 2. Demographics and clinical characteristics of TB patients } \\
\hline \multicolumn{7}{c}{ Time on } \\
dialysis, \\
Patient
\end{tabular}

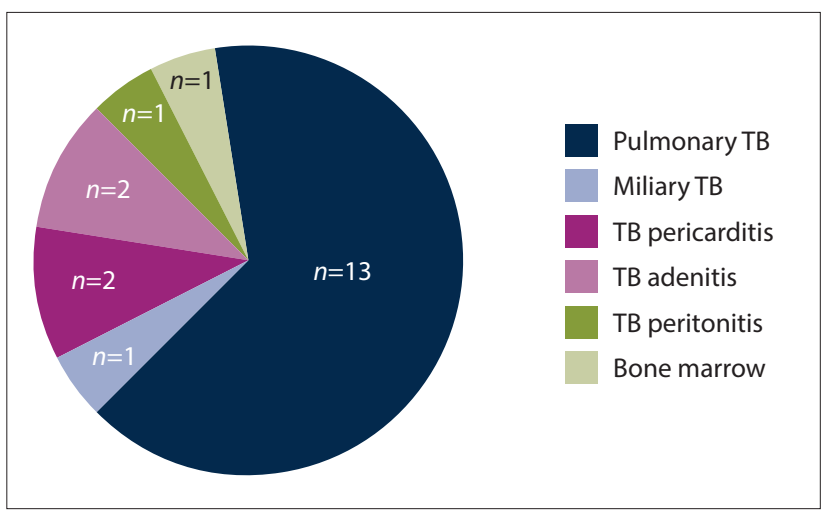

Fig. 1. TB-positive cases: spread of TB diagnosis. $(T B=$ tuberculosis. $)$

bone marrow biopsy showing caseating granulomas and a mycolytic blood culture that confirmed drug-sensitive TB; 1 patient (\#9) had drug-resistant TB (multidrug-resistant TB (MDR-TB)) on culture; 1 (\#12) had pulmonary TB with positive acid-fast bacilli on sputum microscopy (culture was not available), and 1 (\#13) had pulmonary $\mathrm{TB}$ (suggestive chest radiograph) and was positive for drug-sensitive $\mathrm{TB}$ on sputum culture.

Of the 13 probable TB patients, 10 were diagnosed based on clinical features and lymphocytic pleural effusion and 2 had pericardial effusions with stranding on echocardiography. A pericardial tap in both patients revealed a lymphocyte-predominant picture, while 1 patient had a miliary pattern on chest radiography but a negative culture. The incidence of TB infection is shown in Fig. 2.

\section{Discussion}

This single-centre retrospective cohort study revealed an extraordinarily high incidence of $\mathrm{TB}$ in dialysis patients in the Eastern

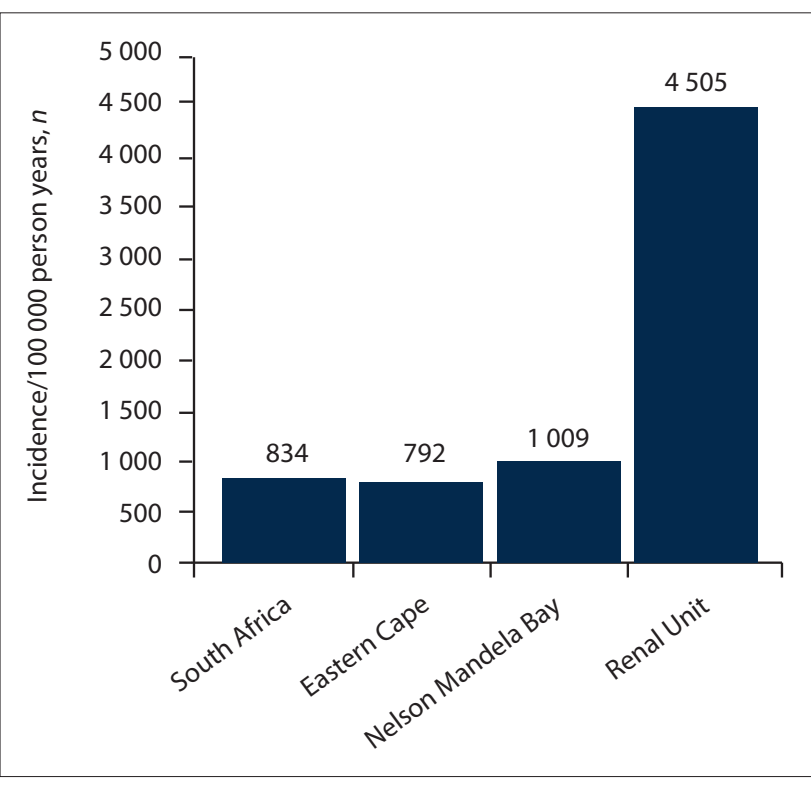

Fig. 2. Tuberculosis incidence in the study population compared with the local, regional and national incidence in 2015. ${ }^{[12,13]}$

Cape, with a calculated incidence 4.1 times that of the local population and $>5$ times that reported in the general population for the country as a whole (Fig. 2). While such a relative risk is consistent with that described in dialysis cohorts from other countries, ${ }^{[8]}$ the absolute risk to our TB patients is extremely high given the very high background prevalence of TB in SA ( 1 in 5 over a 5 -year period). The patients are predominantly young and HIV-negative, reflecting the selection bias in the SA public sector dialysis programme that favours those with less comorbidity. Moosa and Kidd ${ }^{[14]}$ have previously reported on 
the rationing of dialysis treatment in SA - patients are screened and accepted onto the dialysis programme based on favourable medical and socioeconomic criteria. Known TB risk factors that we assessed included age, employment status, smoking, alcohol use, average number of days admitted to hospital per year, housing type, TB contact status, HIV status and vitamin D levels. ${ }^{[15,16]}$ However, of these risk factors, only informal housing and a history of hospitalisation were significantly associated with a diagnosis of TB $(p \leq 0.05)$. In SA, informal housing (shack dwelling) is associated with overcrowding and unhygienic conditions, which would favour the transmission of TB in the community. While a history of hospitalisation may represent a more compromised cohort prone to TB, lack of adequate isolation facilities in our hospital during the study period may have contributed to nosocomial transmission of $\mathrm{TB}$, although this is speculative.

Interestingly, HIV was not predictive of $\mathrm{TB}$, but this is possibly confounded by the use of isoniazid (INH) chemoprophylaxis therapy in this group according to national guidelines and the low overall prevalence of HIV in our unit (11\%). Moreover, all HIV patients on dialysis had undetectable viral loads and relatively preserved CD4+ counts as per dialysis selection criteria discussed above. A higher number of patients with TB had a history of a known TB contact ( $40 \%$ v. $25 \%)$, but this was not statistically significant $(p=0.174)$. The combined vitamin D deficiency/insufficiency prevalence for our cohort was $45.7 \%$ and was not shown to be a significant risk factor for the development of TB. However, vitamin D levels were not measured at the time of TB diagnosis, as the test has not always been available at our study centre.

In our study, the incidence of extrapulmonary TB was very high (30\%) and is consistent with reports from previous studies of CKD patients. ${ }^{[8,11,17-20]}$ The increased tendency for extrapulmonary TB in CKD patients is thought to be due to impaired cellular immunity. ${ }^{[19]}$ In dialysis patients, TB symptoms are often nonspecific and difficult to distinguish from uraemic symptoms, which can result in delayed diagnosis. ${ }^{[8,19]}$ This may also facilitate transmission to other patients, particularly in haemodialysis units, where patients spend much time in close proximity to each other. Of the $20 \mathrm{~TB}$ patients, 7 (35\%) were definite cases, with positive microscopy or culture. Tamayo-Isla et al. ${ }^{[10]}$ in their retrospective TB peritonitis study in SA, reported that TB was isolated in $75 \%$ of their cohort. In our cohort, 13/20 (65\%) patients with TB were culture negative, reflecting the difficulty of definitive TB diagnosis in dialysis patients.

INH chemoprophylaxis in HIV-positive patients has been found to be efficacious in a number of large-scale randomised controlled trials, with up to a $74 \%$ reduction in $\mathrm{TB}$ incidence in select groups displaying a positive tuberculin skin test. ${ }^{[21]}$ There are clear guidelines for this population, but data on INH chemoprophylaxis in CKD patients are lacking. A few randomised clinical trials have assessed INH chemoprophylaxis in both pre- and post-transplant patients and demonstrated its benefit to such patients for up to 1 year. ${ }^{[22-24]}$ Further large randomised clinical studies in the dialysis setting are required to help inform best practice regarding when to commence INH chemoprophylaxis and the most efficacious duration of therapy.

\section{Study limitations and strengths}

There were some limitations to the study. Because of its retrospective nature, we were unable to include dialysis patients who had died during the study period, as their health records were unavailable. We also could not make assumptions regarding hospitalisation as a risk factor for TB, as we could not prove causality. Details regarding the reason for admission, length of stay and timing in relation to onset of TB were not available.

Study strengths include use of a single laboratory with standardised methodology, as well as availability of accurate clinical notes for determination of the diagnosis. While the overall numbers are relatively low for this cohort, a further strength of this study is that it included all living patients on dialysis in the public sector for the entire western part of the Eastern Cape and is therefore inclusive.

\section{Conclusions}

Dialysis patients in the Eastern Cape are at extremely high risk for $\mathrm{TB}$, with a calculated incidence of $4505 / 100000$ population per year. There is a need for improved healthcare structures, including the provision of adequate isolation facilities for TB patients in our hospital and renal unit to reduce transmission. Further research on outcomes and response to TB treatment of CKD patients in SA is required. Whether INH prophylaxis is a reasonable option for TB prevention in this population, remains unknown and should be the subject of further study.

Declaration. The research for this study was done in partial fulfilment of the requirements for SN's MMed (Internal medicine) degree.

Acknowledgements. The authors gratefully acknowledge all the dialysis patients at Livingstone Hospital, Port Elizabeth, SA, who gave of their time and willingly participated in the study.

Author contributions. SN designed the study, collected the data and wrote the manuscript. RF and IO were supervisors and contributed to conceptualising the study, interpreting the data and reviewing the final manuscript. HC performed the statistical analysis. JB and GC contributed to the study design and final manuscript. All authors read and approved the final manuscript.

Funding. None.

Conflicts of interest. None.

World Health Organization. Global Tuberculosis Report 2018. Geneva: WHO, 2018:1-277.

2. Massyn N, Peer N, Padarath A, et al. District Health Barometer 2014/15. Durban: Health Systems Trust, 2015.

3. Meyer TW, Hostetter TH. Uremia. N Engl J Med 2007;357(13):1316-1325. https://doi.org/10.1056/ nejmra071313

4. Dervisoglu E, Yilmaz A, Sengul E. The spectrum of tuberculosis in dialysis patients. Scand J Infect Dis 2006;38:1040-1044. https://doi.org/10.1080/00365540600871044

5. Christopoulos AI, Diamantopoulos A, Dimopoulos PA, et al. Risk factors for tuberculosis in dialysis patients: A prospective multi-center clinical trial. BMC Nephrol 2009;10:36. https://doi. org/10.1186/1471-2369-10-36

6. Unsal A, Ahbap E, Basturk T, et al. Tuberculosis in dialysis patients: A nine-year retrospective analysis. J Infect Dev Ctries 2010;7(3):208-213. https://doi.org/10.3855/jidc.2664

Chia S, Karim M, Elwood RK, et al. Risk of tuberculosis in dialysis patients: A population-based study. Chia S, Karim M, Elwood RK, et al. Risk of

Int J Tuberc Lung Dis 1998;2(12):989-991.
8. Hussein MM, Mooij JM, Roujouleh H. Tuberculosis and chronic renal disease. Semin Dialysis 2003;16(1):38-44. https://doi.org/10.1046/j.1525-139x.2003.03010.x
. Hussein MM, Mooij JM, Roujouleh H. Tuberculosis and chron

9. Erkoc R, Dogan E, Sayarlioglu H, et al. Tuberculosis in dialysis patients, single centre experience from an endemic area. Int J Clin Pract 2004;58(12):1115-1117. https://doi.org/10.1111/j.17421241.2004.00212.x

10. Tamayo-Isla RA, de la Cruz MC, Okpechi IG. Mycobacterial peritonitis in CAPD patients in Limpopo: A 6-year cumulative report from a single center in South Africa. Perit Dial Int 2016;36(2):218-222. https://oi.org/10.3747/pdi.2014.00322

11. World Health Organization. Treatment of Tuberculosis: Guidelines. 4th ed. Geneva:WHO, 2009:23-28.

12. Eastern Cape Provincial AIDS Council. Annual Progress Report 2014/15. 2016. http:// sanac.org.za/download/563/resources/3390/eastern-cape-annual-progress-report_final.pdf (accessed 20 December 2016).

13. World Health Organization. Global Tuberculosis Report 2015. Geneva: WHO, 2015:1-115.

14. Moosa MR, Kidd M. The dangers of rationing dialysis treatment: The dilemma facing a developing

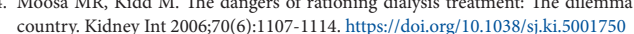

15. Narasimhan P, Wood J, Macintyre CR, et al. Risk factors for tuberculosis. Pulm Med 2013;2013:1-11. . Narasimhan P, Wood J, Macintyre CR,
https://doi.org/10.1155/2013/828939

16. Klote MM, Agodoa LY, Abbott KC. Risk factors for Mycobacterium tuberculosis in US chronic dialysis patients. Nephrol Dial Transpl 2006;21(11):3287-3292. https://doi.org/10.1093/ndt/gfl488

17. Shigidi M, Farouk N, Abulikailik R, et al. Active tuberculous infection among adult Sudanese patients on long term peritoneal dialysis. Arab J Nephrol Transplant 2012;5(3):135-140. 
18. El Kabbaj D, Bahadi A, Oualim Z. Prevalence of tuberculosis in hemodialysis patients. Saudi J Kidney Dis Transpl 2010;21(1):164-167.

19. Sen $\mathrm{N}$, Turunc T, Karatasli $\mathrm{M}$, et al. Tuberculosis in patients with end-stage renal disease undergoing dialysis in an endemic region of Turkey. Transplant Proc 2008;40(1):81-84. https://doi.org/10.1016/j. transproceed.2007.12.003

20. Vikrant S. Tuberculosis in dialysis: Clinical spectrum and outcome from an endemic region. Hemodialysis Int 2018:10-14. https://doi.org/10.1111/hdi.12693

21. Samandari T, Agizew T, Nyirenda S, et al. 6-month versus 36-month isoniazid preventive treatment for tuberculosis in adults with HIV infection in Botswana: A randomised, double-blind, placebo-controlled trial. Lancet Infect Dis 2011;377:1588-1598. https://doi.org/10.1016/s0140-6736(11)60204-3
22. Currie AC, Knight SR, Morris PJ. Tuberculosis in renal transplant recipients: The evidence for prophylaxis. Transplantation 2010;90(7):695-704. https://doi.org/10.1097/tp.0b013e3181ecea8d

23. Vikrant S, Agarwal SK, Gupta S, et al. Prospective randomized control trial of isoniazid chemoprophylaxis during renal replacement therapy. Transpl Infect Dis 2005;7(3-4):99-108. https://doi. org/10.1111/j.1399-3062.2005.00103.x

24. Naqvi R, Naqvi S, Akhtar S, et al. Use of isoniazid chemoprophylaxis in renal transplant recipients. Nephrol Dial Transplant 2010;25:634-637. https:// doi.org/10.1093/ndt/gfp489

Accepted 30 September 2019. 